\title{
Structural and chemical properties of superconducting rare-earth barium copper oxide/BaHfO3 nanocomposites with rare-earth mixtures
}

\author{
Lukas Grünewald $^{1}$, Pablo Cayado ${ }^{2}$, Jens Hänisch ${ }^{2}$, Bernhard Holzapfel ${ }^{2}$ and Dagmar Gerthsen ${ }^{3}$
}

${ }^{1}$ Karlsruhe Institute of Technology (KIT), Laboratory for Electron Microscopy (LEM), Engesserstraße 7, 76131 Karlsruhe, Germany, United States, ${ }^{2}$ Karlsruhe Institute of Technology (KIT), Institute for Technical Physics (ITEP), Hermann-von-Helmholtz-Platz 1, 76344 Eggenstein-Leopoldshafen, Germany, United States, ${ }^{3}$ Laboratorium fürElektronenmikroskopie, KarlsruherInstitutfürTechnologie (KIT), Engesserstr. 7, 76131 Karlsruhe, Germany, United States

The rare-earth barium copper oxides $\mathrm{REBa}_{2} \mathrm{Cu}_{3} \mathrm{O}_{7-\delta}(\mathrm{REBCO}$, with rare earth elements like $\mathrm{Y}$ or $\mathrm{Gd})$ belong to a class of high-temperature superconductors with potential applications as coated conductors for power applications or high-field magnets [1]. Even though $\mathrm{Y}$ is probably the most well-studied RE in REBCO due to the phase stability of YBCO, other REs (e.g.GdBCO) also show promising superconducting properties, albeit with increased difficulty in fabrication. For technical applications, further improvements of the superconducting properties, e.g. the increase of the critical current density, are desired. The latter can be achieved by introducing additional flux-pinning centers, i.e. various structural defects, e.g. non-superconducting $\mathrm{BaHfO}_{3}(\mathrm{BHO})$ nanoparticles. Also, some studies show an enhancement of the critical current density for RE mixtures [2,3] compared with pristine REBCO films. To better understand the influence of RE mixtures on the REBCO microstructure, we have studied the chemical and structural properties of REBCO films with different RE mixtures by analytical scanning transmission electron microscopy (STEM).

The REBCO films were grown on $\mathrm{SrTiO}_{3}(001)$ substrates by chemical solution deposition as described in [4]. Cross-section samples for STEM were prepared by focused-ion-beam milling and in-situ lift-out in an FEI Strata 400S dual-beam system. High- and low-angle annular dark-field (HAADF/LAADF-) STEM in an FEI Titan ${ }^{3} 80-300$ operated at $300 \mathrm{keV}$ is used to characterize the microstructure. Chemical analyses are performed by electron energy-loss spectroscopy (EELS) and energy-dispersive x-ray spectroscopy (EDXS). For EDXS, an FEI Tecnai Osiris equipped with ChemiSTEM technology operated at $200 \mathrm{keV}$ is used.

Experimental results for a (Y,Gd,Dy,Ho,Er) $\mathrm{Ba}_{2} \mathrm{Cu}_{3} \mathrm{O}_{7-\delta}+12 \mathrm{~mol} \% \mathrm{BHO}$ film are presented in the following. Fig. 1(a) shows an overview LAADF-STEM image of the film. The roundish precipitates in the image are mostly BHO nanoparticles. A few extended stacking faults are visible as dark horizontal lines. HAADF-STEM imaging at higher magnification reveals small (few $\mathrm{nm}$ ) strained regions with locally increased $c$ lattice parameter (Fig. 1(b)). The amount of strain of a region in (b) is quantified in (c) and (d). The atom positions of the marked RE sites are determined by Gaussian fitting with Atomap [5] and the RE-plane spacing was evaluated and plotted in (d). Roughly $7.3 \%$ out-of-plane strain is measured at this defect. Moreover, a slightly higher HAADF-STEM intensity is visible at the defect, indicating a possible correlation of local RE content and associated strain. Such defects could act as efficient pinning centers as their size is comparable to the superconducting coherence length of $\sim 2 \mathrm{~nm}$ for REBCO. Ongoing studies will clarify if TEM preparation or measurement artifacts could also cause such defects.

Multivariate statistical analysis algorithms such as non-negative matrix factorization (NMF) can be used for efficient data analysis in EM [6]. Here, we apply NMF using HyperSpy [7] to analyze a STEM-EDXS dataset. The NMF algorithm attempts to separate different (overlapping) chemical phases and (if 
successful) returns a spatial distribution (score map) and a corresponding EDXS spectrum ("loading") for a user-specified number of components, e.g., by the scree-plot method [8] using principal component analysis. Here, NMF is used to separate signals of the embedded BHO nanoparticles within the REBCO film. Fig. 2(a) shows an HAADF-STEM overview image of the mapped region and three (of in total seven) selected chemical phases as determined by NMF. From their spatial distribution, the phases are identified as REBCO matrix (NMF \#0), BHO nanoparticles (NMF \#3), and REs (NMF \#6). (b) and (c) show the corresponding NMF spectra for comparison in selected $\mathrm{x}$-ray energy ranges. $\mathrm{Cu}$ and Ni signals are found in all shown phases due to spurious X-rays from the TEM grid and microscope hardware. NMF \#0 (blue spectrum) shows Ba and RE signals as expected for the REBCO matrix. NMF \#3 (green spectrum) contains signals from all REs ( $\mathrm{Y}$ not shown) with complete depletion of Ba, confirming RE-rich precipitates in the film. The corresponding score map (NMF \#6 in Figure 2(a)) shows a RE-rich precipitate near the substrate interface. These RE-rich precipitates occur with a lower number density than BHO particles. NMF \#3 (orange spectrum) is interpreted as BHO and mostly shows the expected x-ray lines (Ba and Hf). However, a slight Er signal is visible, hinting at preferential mixing of Er in BHO compared to otherREs. Results from another sample with the composition $\left(\mathrm{Y}_{0.33} \mathrm{Er}_{0.33 H o 0.33}\right) \mathrm{BCO}$ will also be shown.

We have shown in this work that advanced processing of analytical TEM data is able to solve a problem that is often encountered in nanoscaled thin films containing several phases. By application of principal component analysis and non-negative matrix factorization, the separation of different phases with dimensions that are significantly smaller than the specimen thickness is possible. 

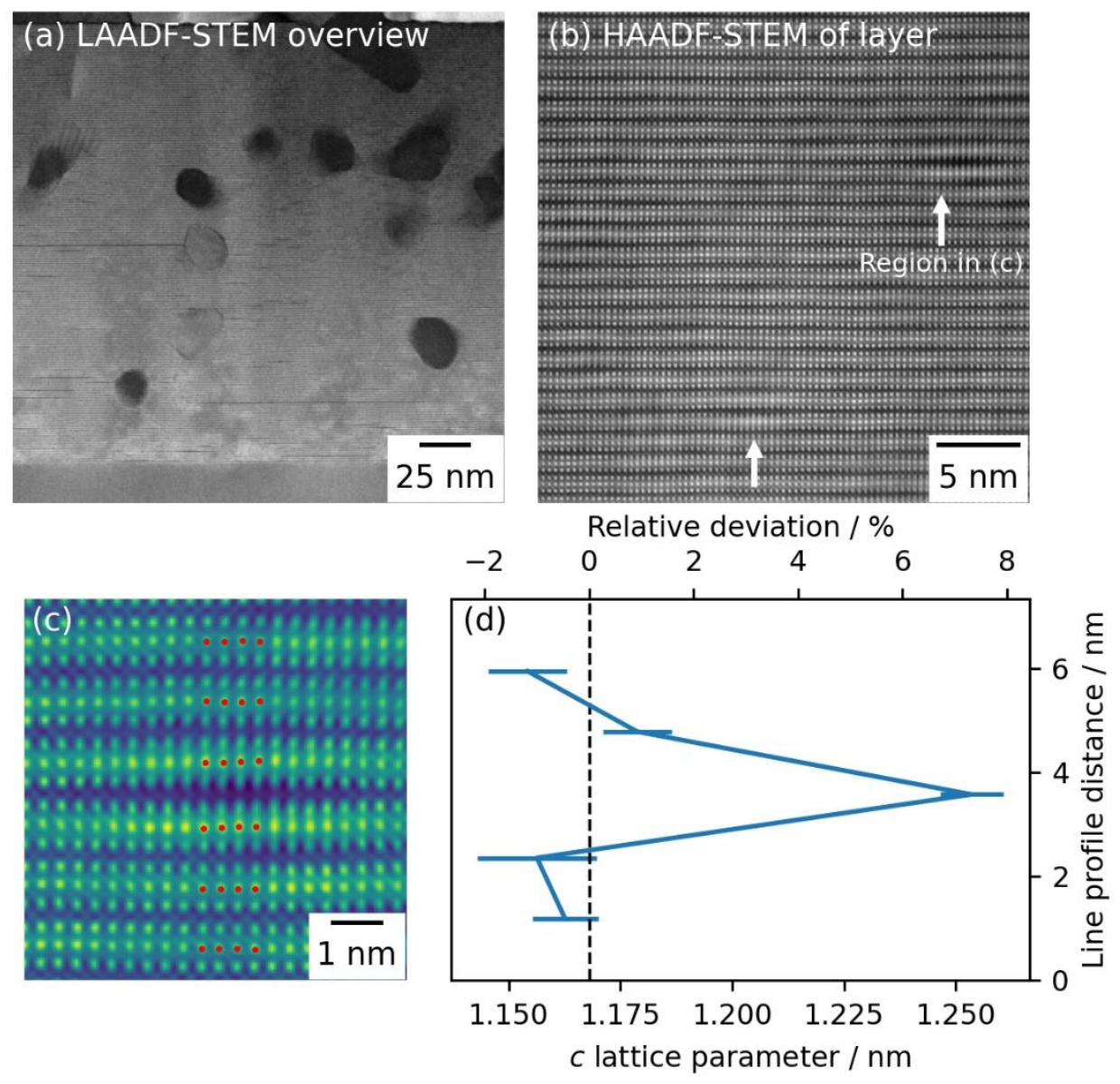

Figure 1. Figure 1: (a) Low-angle ADF-STEM cross-section overview image of a (Y,Gd,Dy,Ho,Er)BCO +12 mol\% BHO nanocomposite film. The dark, roundish particles are mostly BHO. The dark horizontal lines are stacking faults. (b) HAADF-STEM imaging at higher magnification reveals nm-sized regions with local changes in c lattice parameter (marked by arrows). (c, d) Strain analysis of the region marked in (b) shows a $7.3 \%$ out-of-plane strain relative to the literature value of cYBCO $=1.168 \mathrm{~nm}$ (dashed line). A slightly higher HAADF Z-contrast is observed at the RE sites (marked in red in (c)) around the strained region.

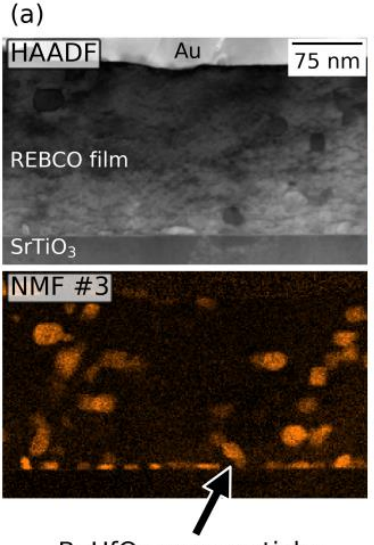

$\mathrm{BaHfO}_{3}$ nanoparticles

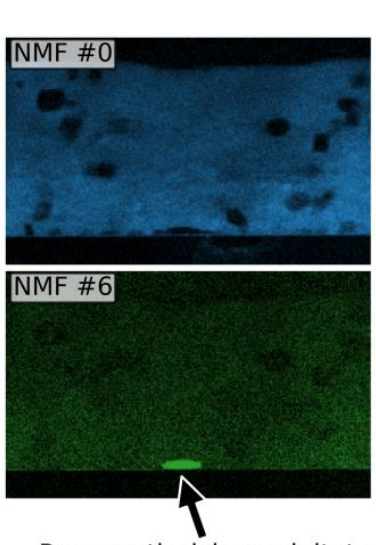

Rare-earth rich precipitate

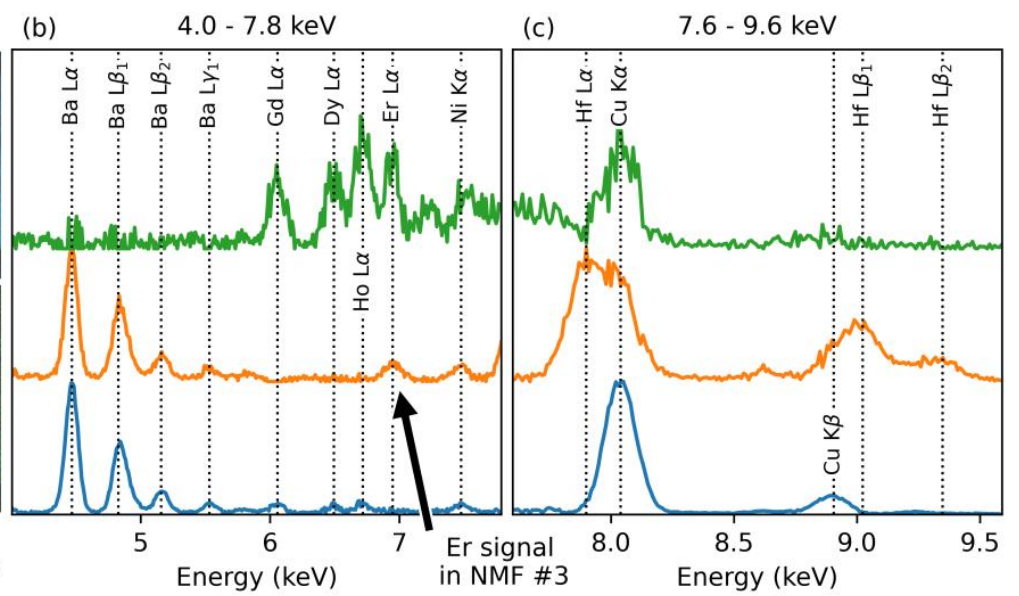


Figure 2. Figure 2: (a) HAADF image and color-coded NMF score maps and (b,c) corresponding loadings of a STEM-EDXS dataset from selected x-ray energy ranges. The identified x-ray lines are marked in (b) and (c). $\mathrm{Cu}$ stems from REBCO and the TEM grid. Ni is a spurious signal from the microscope. NMF \#0 (blue color) is identified as the main REBCO phase with strong $\mathrm{Ba}, \mathrm{Cu}$, and small RE signals. (b) NMF \#6 (green color) shows a high concentration of all REs and Ba depletion. The spatial distribution of NMF \#6 in (a) shows a RE-rich precipitate. NMF \#3 (orange color) shows increased (c) Hf and (b) Ba signals and is identified as BHO nanoparticles. (b) A small Er signal is visible in NMF \#3, stemming from a possible mixing of Er with BHO.

References

[1] X. Obradors, T. Puig, Supercond. Sci. Technol.27 (2014) 044003.

[2] J.L. MacManus-Driscoll et al., Appl. Phys. Lett.84 (2004) 5329-5331.

[3] P. Cayado et al., RSC Adv.8 (2018) 42398-42404.

[4] M. Erbe et al., J. Mater. Chem. A2 (2014) 4932-4944.

[5] M. Nord et al., Adv. Struct. Chem. Imaging3 (2017) 9.

[6] S. Muto, M. Shiga, Microscopy69 (2020) 110-122.

[7] F. de la Peña et al., Microsc. Microanal.23 (2017) 214-215.

[8] R.B. Cattell, Multivar. Behav. Res. 1 (1966) 245-276. 